\title{
Evaluation of Current Heparin Weight Based Protocol in Obese Patients
}

\author{
Marie Lafosse \\ Joe DiMaggio Children's Hospital, Hollywood, Florida, marie.lafosse@flhosp.org \\ Simon Leung \\ Memorial Regional Hospital, Hollywood, Florida, sleung@mhs.net \\ Carla Hawkins \\ Memorial Regional Hospital, Hollywood, Florida, chawkins@mhs.net \\ Madeline Camejo \\ Memorial Regional Hospital, Hollywood, Florida, mcamejo@mhs.net
}

Follow this and additional works at: https://nsuworks.nova.edu/ijahsp

Part of the Pharmacy and Pharmaceutical Sciences Commons

\section{Recommended Citation}

Lafosse M, Leung S, Hawkins C, Camejo M. Evaluation of Current Heparin Weight Based Protocol in Obese Patients. The Internet Journal of Allied Health Sciences and Practice. 2016 Jan 01;14(1), Article 7.

This Manuscript is brought to you for free and open access by the College of Health Care Sciences at NSUWorks. It has been accepted for inclusion in Internet Journal of Allied Health Sciences and Practice by an authorized editor of NSUWorks. For more information, please contact nsuworks@nova.edu. 


\title{
Evaluation of Current Heparin Weight Based Protocol in Obese Patients
}

\begin{abstract}
Purpose: To evaluate the difference in achieving goal activated partial thromboplastin time (aPTT) within 24 hours utilizing the institutional heparin weight based protocol between obese and non-obese patients. Methods: Retrospective, non-randomized, open label chart review in a community based hospital. Patients age 18 years or older receiving heparin therapy for greater than or equal to 24 hours identified. Patients were excluded if the protocol was utilized for acute coronary syndrome or interrupted within the initial 24 hours of therapy. Patients were also excluded if any deviations from the protocol were identified. The primary endpoint evaluated the difference in achieving goal aPTT within 24 hours between obese and non-obese patients. The secondary endpoint evaluated the difference in achieving goal aPTT within 24-48 hours and safety outcomes such as bleeds. Results: Of the 253 patients identified, 167 patients were included and 86 patients were excluded. Seventy-two (71.3\%) of the nonobese and $48(72.7 \%)$ of obese patients achieved goal aPTT within 24 hours (odd ratio [OR]: 0.98, $95 \%$ confidence interval (Cl): 0.6 to 1.58). Sixteen (15.8\%) of nonobese and $13(19.7 \%)$ of the obese patients achieved goal aPTT within 24 to 48 hours (OR: $0.77,95 \% \mathrm{Cl}: 0.3$ to 1.95$)$. Two $(1.9 \%)$ of the nonobese and $3(4.5 \%)$ of the obese patients had a documented major bleed (OR: 0.42 and $95 \% \mathrm{Cl}: 0.69$ to 2.61). Conclusion: Overall, there was no difference in achieving goal aPTT between nonobese and obese patients while utilizing our facility's weight based heparin dosing. No major bleeding was documented that warranted discontinuation of therapy, and bleeding incidences between both groups were similar.
\end{abstract}

\section{Author Bio(s)}

- Marie Lafosse, Pharm.D., BCPS is a Clinical Pharmacist at Joe DiMaggio Children's Hospital in Hollywood, Florida.

- Simon Leung, MS, Pharm.D., BCPS, is a Clinical Coordinator in the Department of Pharmacy at Memorial Regional Hospital in Hollywood, Florida.

- Carla Hawkins, Pharm.D., BCPS, is a Clinical Coordinator in the Department of Pharmacy at Memorial Regional Hospital in Hollywood, Florida.

- Madeline Carmejo, MS, Pharm.D., is the Director of Pharmacy at Memorial Regional Hospital in Hollywood, Florida.

\section{Acknowledgements}

The authors gratefully acknowledge Dr. Angel Maldonado, Dr. Michael Nash, and Monica Lafosse for their statistical and editorial support. 


\title{
TIJAHSP \\ The Internet Journal of Allied Health Sciences and Practice
}

Dedicated to allied health professional practice and education

Vol. 14 No. 1 ISSN 1540-580X

\section{Evaluation of Current Heparin Weight Based Protocol in Obese Patients}

\author{
Marie Lafosse, Pharm.D., BCPS1 \\ Simon Leung, MS, Pharm.D., BCPS2 \\ Carla Hawkins, Pharm.D., BCPS² \\ Madeline Camejo, MS, Pharm.D. ${ }^{2}$
}

1. Department of Pharmacy, Joe DiMaggio Children's Hospital, Hollywood, FL.

2. Department of Pharmacy, Memorial Regional Hospital, Hollywood, FL.

United States

\begin{abstract}
Purpose: To evaluate the difference in achieving goal activated partial thromboplastin time (aPTT) within 24 hours utilizing the institutional heparin weight based protocol between obese and non-obese patients. Methods: Retrospective, non-randomized, open label chart review in a community based hospital. Patients age 18 years or older receiving heparin therapy for greater than or equal to 24 hours identified. Patients were excluded if the protocol was utilized for acute coronary syndrome or interrupted within the initial 24 hours of therapy. Patients were also excluded if any deviations from the protocol were identified. The primary endpoint evaluated the difference in achieving goal aPTT within 24 hours between obese and non-obese patients. The secondary endpoint evaluated the difference in achieving goal aPTT within 24-48 hours and safety outcomes such as bleeds. Results: Of the 253 patients identified, 167 patients were included and 86 patients were excluded. Seventy-two (71.3\%) of the nonobese and $48(72.7 \%)$ of obese patients achieved goal aPTT within 24 hours (odd ratio [OR]: 0.98, $95 \%$ confidence interval (CI): 0.6 to 1.58). Sixteen (15.8\%) of nonobese and $13(19.7 \%)$ of the obese patients achieved goal aPTT within 24 to 48 hours (OR: $0.77,95 \% \mathrm{Cl}: 0.3$ to 1.95$)$. Two (1.9\%) of the nonobese and $3(4.5 \%)$ of the obese patients had a documented major bleed (OR: 0.42 and $95 \%$ Cl: 0.69 to 2.61). Conclusion: Overall, there was no difference in achieving goal aPTT between nonobese and obese patients while utilizing our facility's weight based heparin dosing. No major bleeding was documented that warranted discontinuation of therapy, and bleeding incidences between both groups were similar.
\end{abstract}

\section{BACKGROUND}

Unfractionated heparin (UFH) is an indirect factor Xa and lla inhibitor within the clotting cascade, preventing the formation of fibrin clots. ${ }^{1}$ Its anticoagulant effect is often unpredictable due to its large molecular weight (up to $30,000 \mathrm{Da}$ ), unique pharmacological profile, and binding activity to plasma proteins. ${ }^{1}$ In order to overcome the unpredictable effect of UFH, though it is labor intensive, activated partial thromboplastin time (aPTT) with a recommended goal between 1.5 to 2.5 times the baseline has been used to determine the appropriateness of current therapy. ${ }^{1-3}$ Currently UFH has proven beneficial when preventing thromboembolic disease, but its efficacy is dependent on appropriate dosing. Raschke et al performed a randomized controlled trial that compared weight based UFH dosing with the standard nomogram. ${ }^{4}$ At 24 hours, $97 \%$ of participants in the weight-based compared to $77 \%$ in the standard nomogram achieved goal aPTT. ${ }^{4}$

Higher plasma concentrations of prothrombotic factors have been identified in obese patients. ${ }^{5}$ Abdominal obesity has shown to correlate with an increased mean platelet volume. This leads to increased adhesiveness and hyperaggregability, suggesting higher risk for systemic thrombosis. ${ }^{6}$ In a retrospective setting, evaluating any potential effects of body mass index (BMI) on aPTT levels in cardiac patients found no significant difference $(p=0.5)$ in frequency of first aPTT level within range $\left(60-90\right.$ secs). ${ }^{7}$ According to the 2012 American College of Chest Physician guidelines for treatment of venous thromboembolism, recommendations for UFH therapy include an initial bolus dose of 80 units $/ \mathrm{kg}$ followed by an initial infusion of $18 \mathrm{units} / \mathrm{kg} / \mathrm{hour}$; this therapy helps target an aPTT level of $1.5-2.5$ times the baseline aPTT.1,8,9 Currently, a universal UFH weight-based nomogram does not exist and information on the dosing of UFH and efficiency of an UFH nomogram in obese patients is lacking.

(c) The Internet Journal of Allied Health Sciences and Practice, 2015 
Although each hospital has adopted its own nomogram with a target aPTT level and dose titration based on the repeat measure of aPTT levels, clinical data targeting if heparin weight-based nomogram would have the similar effects between obese and nonobese patients is limited. Spruill et al found no difference in time to target aPTT between nonobese and obese patients when evaluating the need for different heparin dosing between the two groups. ${ }^{10}$

Our institutional heparin weight-based protocol for standard dosing utilizes a bolus of 65 units/kg (maximum 5,000 units) and an initial infusion rate of 14 units $/ \mathrm{kg} / \mathrm{hr}$ (maximum 15 units/hour) with standardized dose adjustment based on repeat measures of aPTT values. Standard dosing is utilized in patients with an indication other than acute coronary syndrome (ACS). These indications include, but are not limited to, atrial fibrillation/flutter, deep vein thrombosis, pulmonary embolism, ischemic stroke/transient ischemic attack, heart valve disorder, peripheral vascular disease, pulmonary hypertension, and cardiomyopathy. Our facility adopted the more conservative approach from the 2012 ACCP guideline recommendation of administering 60-70 units/kg (maximum 5,000 units) followed by an infusion of 12-15 units/kg/hr for safety purposes. ${ }^{1}$ An inadequate UFH bolus and/or initial continuous infusion has been shown to delay reaching goal aPTT for $>24$ hours in obese $\left(\mathrm{BMI} \geq 30 \mathrm{~kg} / \mathrm{m}^{2}\right)$ patients with venous thromboembolism. ${ }^{11}$ Our institution's UFH dosing approach has not been rigorously evaluated and conflicting data exist on achieving target aPTT between nonobese and obese patients. ${ }^{10,11}$ Therefore, this study is designed to evaluate the difference in achieving goal aPTT within 24 hours between obese and non-obese patients with our current heparin weight-based protocol.

\section{METHODS}

This retrospective, non-randomized, open label chart review was conducted at a large community base teaching hospital. Data collection began after Institutional Review Board approval. The principal investigator performed all data collection. Patients were identified through the institutional electronic medical records between August 1, 2011, and August 1, 2013, using the standard UFH 25,000 units mixed in $250 \mathrm{~mL}$ of dextrose $5 \%$ (D5W) premix product. Information obtained from the institutional electronic medical record were transferred to the data collection tool including demographic data, weight, height, indication for UFH, appropriate management of protocol based on aPTT levels (time of each UFH bolus, rate change and time aPTT drawn), pertinent lab values/tests and progress notes to assess major bleeding. The data were later transferred to a spreadsheet for secured storage.

Eligible patients included those at least 18 years of age that utilized standard dosing heparin weight-based protocol for $\geq 24$ hours. Exclusion criteria included those who utilized the protocol for ACS or if therapy was interrupted within the initial 24 hours. Incorrect usage of the protocol such as omission of initial bolus dose and/or incorrect dose change based on aPTT level resulted in exclusion of that patient.

The primary endpoint of this study evaluated the difference in achieving goal aPTT within 24 hours between obese and nonobese patients utilizing our institutional heparin weight-based protocol (Figure 1). Secondary endpoint evaluated the difference in achieving goal aPTT within 24 to 48 hours between obese and non-obese patients. Based on the World Health Organization, the non-obese patients were identified as those with a BMl $<30 \mathrm{~kg} / \mathrm{m}^{2}$ and obese patients with BMl $\geq 30 \mathrm{~kg} / \mathrm{m}^{2} .{ }^{12} \mathrm{Goal}$ aPTT refers to achieving 1.5 to 2.5 times the baseline aPTT level determined by the photometric assay and BCS instrument. In reference to our institutional protocol, goal aPTT is between 50-70 seconds. Safety endpoint according to the International Society of Thrombosis and Haemostasis (ISTH) assessed major bleeding, any fatal bleeding, and/or symptomatic bleeding in a critical area or organ (intracranial, intraspinal, intraocular, retroperitoneal, intraarticular or pericardial), and bleeding causing a fall in hemoglobin level $\geq 2 \mathrm{~g} / \mathrm{dL}$ and/or leading to transfusion of $\geq 2$ units of whole blood or red cells. ${ }^{13}$

(C) The Internet Journal of Allied Health Sciences and Practice, 2015 


\begin{tabular}{|c|c|c|}
\hline Patient's PTT & Action & Order aPTT \\
\hline Initial dose & $\begin{array}{c}65 \text { units } / \mathrm{kg} \text { bolus ( } \max 5,000 \text { units), then } 14 \\
\text { units } / \mathrm{kg} / \mathrm{hr}(\max 15 \mathrm{ml} / \mathrm{hr})\end{array}$ & 6 hours after starting heparin \\
\hline $\mathrm{aPTT}<40 \mathrm{~s}$ & $\begin{array}{c}65 \text { units } / \mathrm{kg} \text { bolus (max } 5,000 \text { units), add } 2 \mathrm{ml} \text { to } \\
\text { current rate }\end{array}$ & 6 hours after bolus \\
\hline aPTT, 40-49s & 20 units $/ \mathrm{kg}$ bolus, add $1 \mathrm{ml}$ to current rate & 6 hours after bolus/rate change \\
\hline aPTT, 50-70s & No change & In 12 hours \\
\hline aPTT, 71-85s & Subtract $1 \mathrm{ml}$ from current rate & 6 hours after rate change \\
\hline aPTT, 86-100s & $\begin{array}{c}\text { Stop infusion for one hour and subtract } 2 \mathrm{ml} \text { from } \\
\text { current rate }\end{array}$ & 6 hours after rate change \\
\hline aPTT, 100-150s & $\begin{array}{c}\text { Stop infusion for two hours and subtract } 3 \mathrm{ml} \text { from } \\
\text { current rate }\end{array}$ & 6 hours after rate change \\
\hline $\mathrm{aPTT}>150 \mathrm{~s}$ & 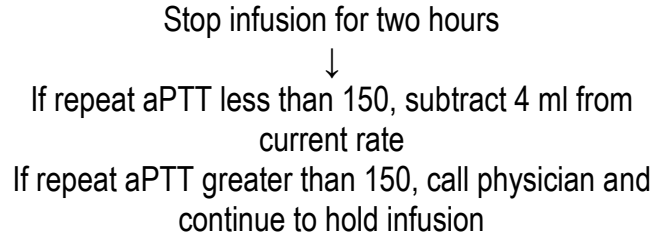 & $\begin{array}{c}\text { STAT aPTT } 2 \text { hours after infusion } \\
\text { stopped } \\
\downarrow \\
\downarrow \\
\text { Repeat aPTT } 6 \text { hours after rate change }\end{array}$ \\
\hline
\end{tabular}

Figure 1: Weight-Based Nomogram

\section{Statistical analysis}

A sample size of 150 patients was needed to detect an estimated observed effect size of 0.5 between the two study groups, with an a of 0.05 , a $\beta$ of 0.2 and a possible $5 \%$ dropout rate. Endpoints were evaluated for the per-protocol population. Statistical evaluation comparing non-parametric and parametric data was accomplished with chi-squared and two sample t-test, respectively. All non-parametric data was analyzed and expressed as an odds ratio (OR) with 95\% confidence interval (Cl). Continuous data was analyzed and presented as descriptive statistics (mean \pm standard deviation). All statistical analysis was analyzed via Number Cruncher Statistical System (NCSS) software. A p-value $<0.05$ was considered statistically significant.

\section{RESULTS}

During the study period, 253 patients were identified based on the utilization of the standard UFH 25,000 units/D5W 250ml premix product. Patient selection is depicted in Figure 2. Based on the study criteria, 167 patients were included and 86 patients were excluded. Of the 167 patients included in the analysis, 101 were in the nonobese and 66 were in the obese group. Baseline characteristics are summarized in Table 1. Except for BMI, the patient characteristics between the two groups did not differ significantly in age, height, and sex.

\section{Table 1. Baseline Patient Characteristics}

\begin{tabular}{|c|c|c|c|}
\hline Group (kg/m²) & $\begin{array}{c}\mathrm{BMI}<30 \\
\mathrm{n}=101(\%)\end{array}$ & $\begin{array}{l}\mathrm{BMI} \geq 30 \\
\mathrm{n}=66(\%)\end{array}$ & $P$ values \\
\hline Age (years) a & $64 \pm 20$ & $58 \pm 15$ & NS \\
\hline Female & $n=43(42 \%)$ & $\mathrm{n}=35(53 \%)$ & NS \\
\hline Male & $\mathrm{n}=58(57 \%)$ & $\mathrm{n}=31(47 \%)$ & NS \\
\hline Height $(\mathrm{cm})^{a}$ & $170 \pm 11$ & $168 \pm 13$ & NS \\
\hline $\begin{array}{c}\text { Indications } \\
\text { DVT } \\
\text { PE } \\
\text { Atrial fibrillation } \\
\text { Valve disorder } \\
\text { PVD } \\
\text { Others }\end{array}$ & $\begin{array}{c}23 \\
22 \\
20 \\
11 \\
4 \\
21\end{array}$ & $\begin{array}{c}14 \\
18 \\
19 \\
8 \\
3 \\
4\end{array}$ & \\
\hline
\end{tabular}

a Results are presented as mean \pm standard deviation

${ }^{b}$ Other indications include cardiomyopathy, hypercoagulable state and ischemic stroke Deep Vein Thrombosis (DVT), Pulmonary Embolism (PE), Peripheral Vascular Disease (PVD) 


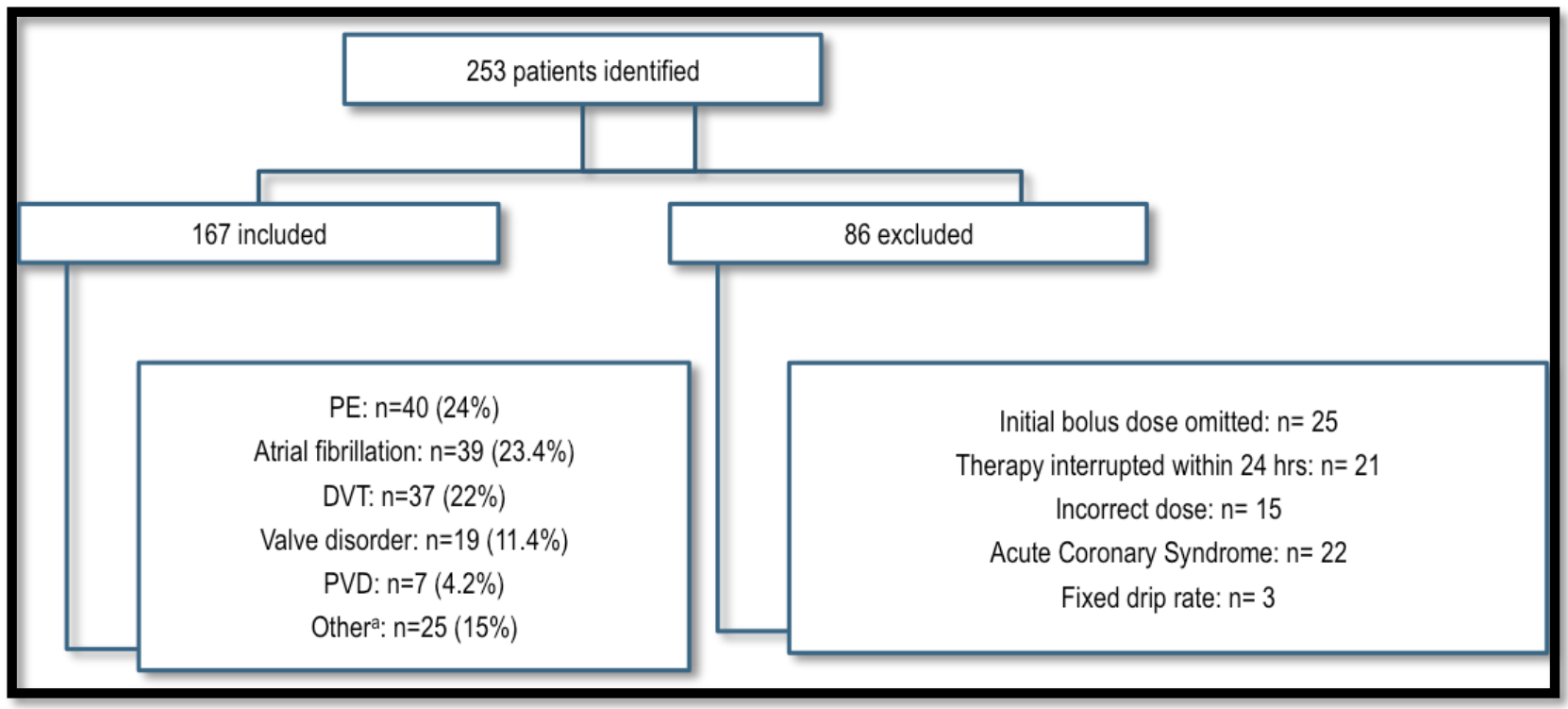

Figure 2: Patient Selection

Deep Vein Thrombosis (DVT), Pulmonary Embolism (PE), Peripheral Vascular Disease (PVD) aOther indications include cardiomyopathy, hypercoagulable state and ischemic stroke

For the primary endpoint, $72(71.3 \%)$ of the nonobese and $48(72.7 \%)$ of obese patients achieved goal aPTT within 24 hours (OR: 0.98 and $95 \% \mathrm{Cl}: 0.6$ to 1.58) (Figure 3) (Table 2). Patients who did not achieve goal aPTT within 24 hours were analyzed for the secondary endpoint of achieving goal aPTT within 24 to 48 hours. The results showed 16 (15.8\%) of non-obese and 13 (19.7\%) of obese patients achieved goal aPTT of 50 to 70 seconds within 24 to 48 hours (OR: 0.77 and $95 \% \mathrm{Cl}: 0.3$ to 1.95 ) (Table 2). Approximately $90 \%$ of patients in both groups achieved goal aPTT within 48 hours (Figure 4).

\begin{tabular}{|c|c|c|c|}
\hline \multicolumn{4}{|c|}{ Table 2. Study Endpoint } \\
\hline & $\begin{array}{c}\mathrm{BMI}<30 \\
\mathrm{n}=101(\%)\end{array}$ & $\begin{array}{l}\mathrm{BMI} \geq 30 \\
\mathrm{n}=66(\%)\end{array}$ & $\begin{array}{c}\text { Odds Ratio } \\
95 \% \text { Confidence Interval }\end{array}$ \\
\hline $\begin{array}{c}\text { Achieved Goal aPTT Within } \\
24 \text { Hours }\end{array}$ & $72(71.3)$ & $48(72.7)$ & $\begin{array}{c}\text { OR: } 0.98 \\
95 \% \mathrm{Cl}: 0.6 \text { to } 1.58\end{array}$ \\
\hline $\begin{array}{c}\text { Achieved Goal aPTT Within } \\
24-48 \text { Hours }\end{array}$ & $16(15.8)$ & $13(19.7)$ & $\begin{array}{c}\text { OR: } 0.77 \\
95 \% \text { Cl: } 0.3 \text { to } 1.95\end{array}$ \\
\hline Major Bleeding & $2(1.9)$ & $3(4.5)$ & $\begin{array}{c}\text { OR: } 0.42 \\
95 \% \text { Cl: } 0.69 \text { to } 2.61\end{array}$ \\
\hline
\end{tabular}

When evaluating the safety endpoint during the course of UFH therapy, two (1.9\%) of the 101 nonobese and three (4.5\%) of the 66 obese patients had a documented major bleed during the specified hospitalization (OR: 0.42 and $95 \% \mathrm{Cl}: 0.69$ to 2.61) (Table 2). All five of the patients were categorically determined to experience major bleeding due to a $\geq 2 \mathrm{~g} / \mathrm{dL}$ drop in hemoglobin requiring $>2$ units of red cells transfusion. One patient in the nonobese group experienced rectal bleeding. Two patients in the obese group were documented to have experienced hematuria during that hospitalization. Overall, no major bleeding was documented that warranted discontinuation of therapy. 


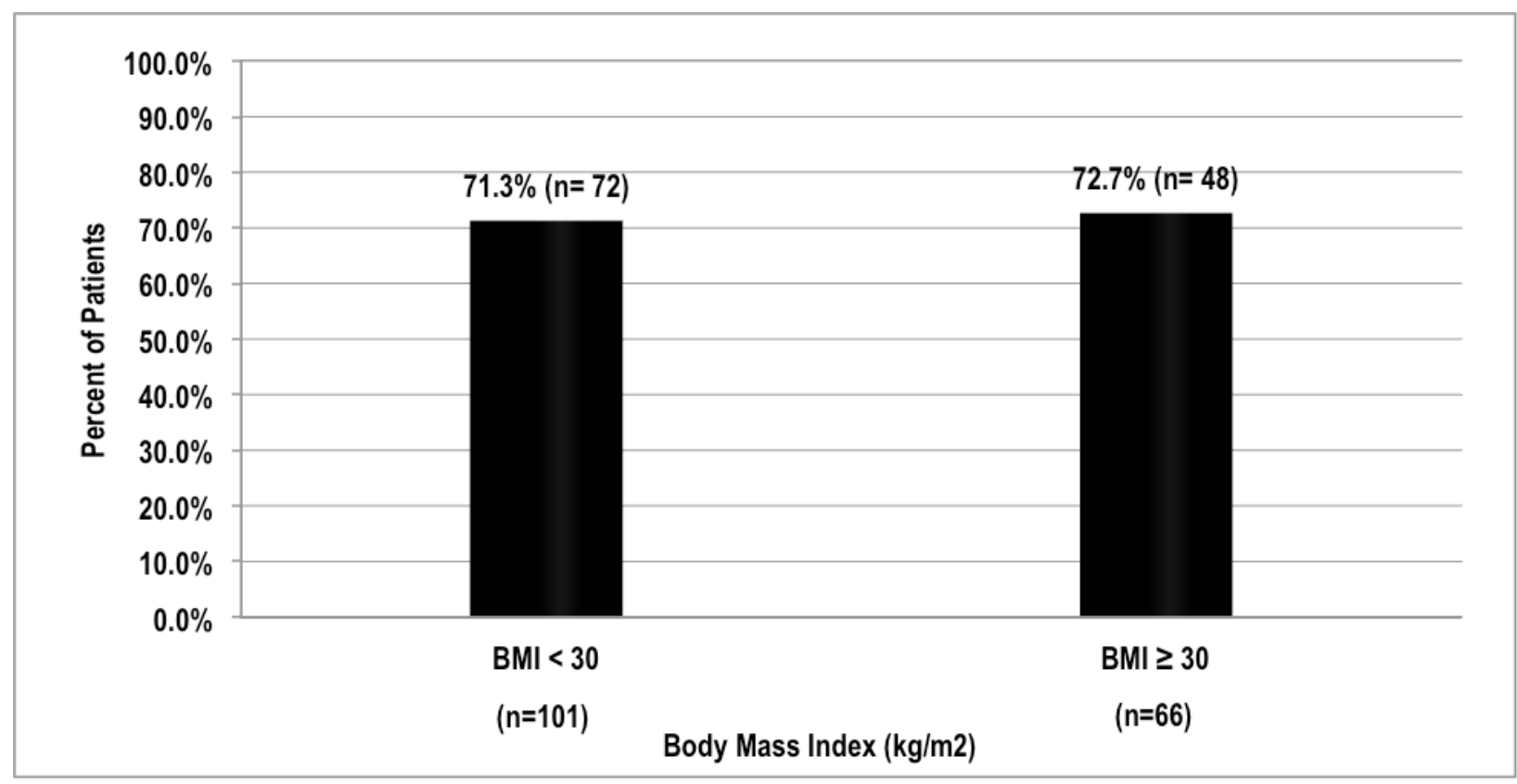

Figure 3: Achieving Goal aPTT Within 24 Hours

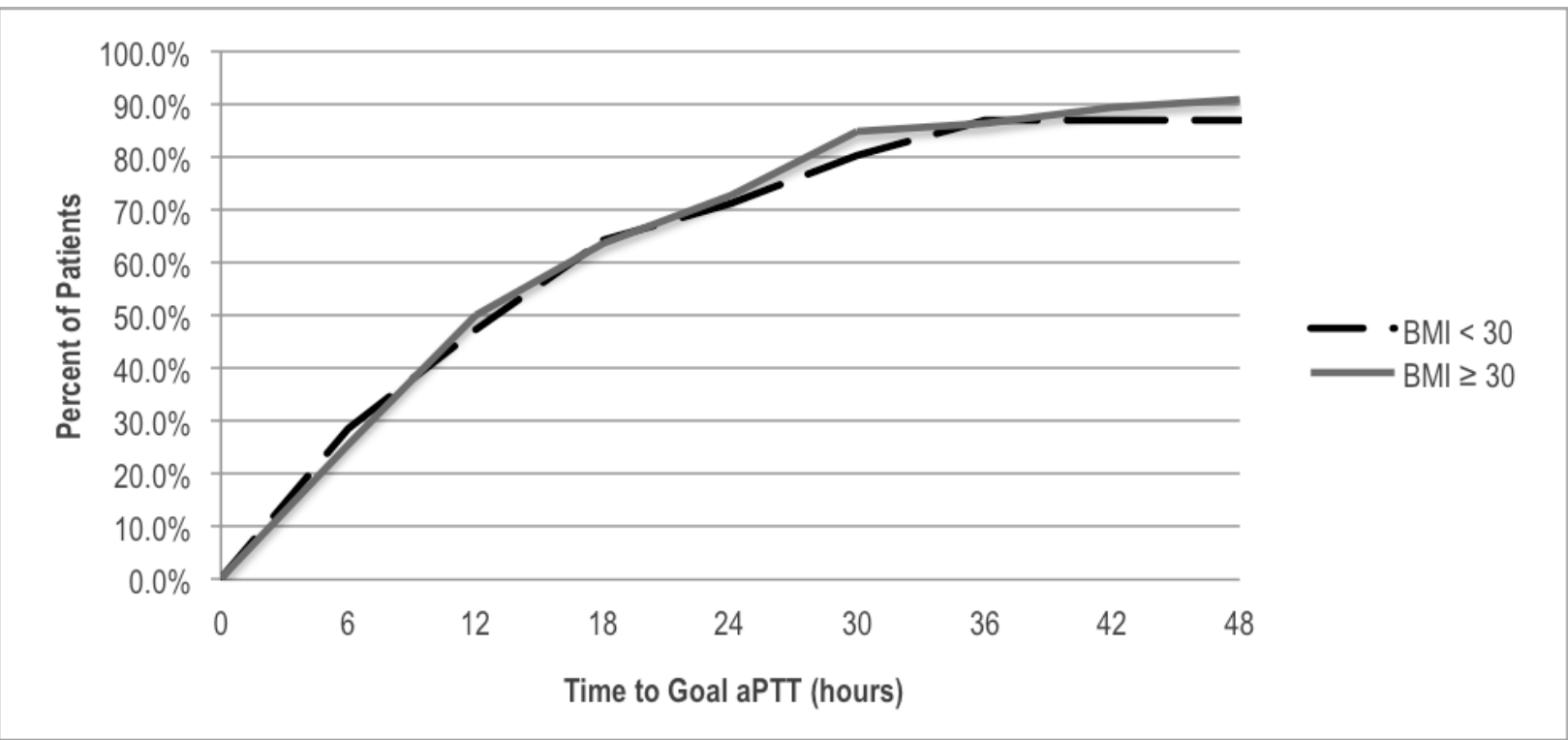

Figure 4: Percent of Patients' Achieving Goal aPTT

\section{Discussion}

To our knowledge, this is the first study to evaluate the heparin weight based protocol in our institution between nonobese and obese patients. Raschke et al compared the performance of a weight based UFH approach ( 80 units $/ \mathrm{kg}$ initial bolus followed by $18 \mathrm{units} / \mathrm{kg} / \mathrm{hr}$ infusion and adjust based on nomogram) for treatment of venous or arterial thromboembolism or for unstable angina. ${ }^{4}$ Study results demonstrated that UFH weight based dosing was superior to the standard of practice consisting of fixed bolus doses and infusion rate in the early 1990 s. ${ }^{4}$ About $97 \%$ and $77 \%$ of patients achieved therapeutic goal within 24 hours, respectively. ${ }^{4}$ However; Raschke et al did not stratify the data based on patients' body weight. Mean weight in both study arms (UFH weight based and standard UFH nomogram) were $80.9 \mathrm{~kg}$ and $76.9 \mathrm{~kg}$, respectively. ${ }^{4} \mathrm{It}$ was difficult to extrapolate the results of this study to obese patients since the body mass indexes were not presented. Our study demonstrated that despite the more conservative weight-based regimen, $72.7 \%$ obese patients and $71.3 \%$ non-obese patients achieved goal aPTT in the initial 
24 hours utilizing our heparin weight-based protocol. ${ }^{9}$ No difference was detected between both study groups within the initial 24 and 48 hours.

While evaluating major bleeding, based on the ISTH definition, a drop of $\geq 2 \mathrm{~g} / \mathrm{dL}$ in hemoglobin was usually detected on day 2 of therapy. Blood products were commonly administered if hemoglobin levels dropped below $8 \mathrm{~g} / \mathrm{dL}$. It is important to mention, of the documented bleeding episodes, none were detected in the critical areas or organs. One nonobese patient with documented bleeding had a past medical history significant for Crohn's Disease which can confound the bleeding events. As with results from previous studies, no significant difference was found between nonobese and obese patient bleeding events. ${ }^{4,7}$ Lower or similar bleeding rates $(1.9 \%$ and $4.5 \%)$ were also detected compared to previous studies $(5.7 \%$ and 4.5$)$ in both nonobese and obese patients, respectively. ${ }^{7}$

With these findings, our institution has revised the weight-based protocol. From a practice standpoint, clinical outcomes include achieving goal aPTT sooner within the 24 hour time span while maintaining safety. The bolus dose and initial infusion rate has been revised reflecting current recommendations from CHEST guidelines. ${ }^{1,8,9}$ However, a maximum cap still applies but has increased to 10,000 units of heparin. With these changes, our institution plans on conducting a further study to determine safety and if a difference in achieving goal aPTT is detected between non-obese and obese patients.

While this study demonstrates equal appropriateness between nonobese and obese patient utilizing the current therapy at our facility, there were some limitations. This protocol was not universal among hospitals demonstrating low external validity. It would be difficult to extrapolate our results and recommendations to other institutions. This was a non-randomized study limiting generalization to all patients in the hospital. Many patients analyzed within 24-48 hours of therapy, did not receive therapy for the entire 48 hours. For some, therapy was discontinued prior to achieving goal aPTT which lowers the percentage of patients achieving goal aPTT within 48 hours. For many patients, aPTT levels were not obtained at the exact time indicated delaying the time to adjust the infusion rate; with subtle delays, achieving aPTT levels may have been affected.

\section{Conclusion}

Overall, there was no difference in achieving goal aPTT between nonobese and obese patients while utilizing our facilities weight- based heparin dosing. Of the study population, $71.3 \%$ of non-obese and $72.7 \%$ of obese patients achieved goal aPTT within 24 hours. Approximately $90 \%$ of patients achieved goal aPTT within 48 hours in both the nonobese and obese group. No major bleeding was documented that warranted discontinuation of therapy. Bleeding incidents between both groups were not significant. Lacking a universal benchmark on timing of reaching aPTT goal, our heparin weight-based program is considered safe and appropriate.

\section{References}

1. Garcia DA, Baglin TP, Weitz J, Samama MM. Parenteral anticoagulants: Antithrombotic therapy and prevention of thrombosis, 9th ed: American College of Chest Physicians Evidence-Based Clinical Practice Guidelines. Chest. 2012 Feb;141(2 Suppl):e24s-43s. [PMID 22315264]

2. Basu D, Gallus A, Hirsh J, Cade J. A prospective study of the value of monitoring heparin treatment with the activated partial thromboplastin time. N Engl J Med. 1972 Aug;287(7):324-7. [PMID 5041701]

3. Smith SB, Geske JB, Maguire JM, Zane NA, Carter RE, Morgenthaler TI. Early anticoagulation is associated with reduced mortality for acute pulmonary thrombus. Chest. 2010 Jun;137(6):1382-90. doi:10.1378/chest.09-0950 [PMID 20081101]

4. Raschke RA, Reilly BM, Guidry JR, Fontana JR, Srinivas S. The weight-based heparin dosing nomogram compared with a "standard care" nomogram. A randomized controlled trial. Ann Intern Med. 1993 Nov 1;119(9):874-81. [PMID 8214998]

5. De Pergola G, Pannacciulli N. Coagulation and fibrinolysis abnormalities in obesity. J Endocrinol Invest. 2002 Nov;25(10):899-904. [PMID 12508953]

6. Targher G, Zoppini G, Moghetti P, Day CP. Disorders of coagulation and hemostasis in abdominal obesity: Emerging role of fatty liver. Semin Thrombo Hemost. 2010 Feb;36(1):41-8. [PMID 20391295]

7. Bauer SR, Ou NN, Dreesman BJ, Armon JJ, Anderson JA, Cha SS, Oyen LJ. Effect of body mass index on bleeding frequency and activated partial thromboplastin time in weight-based dosing of unfractionated heparin: A retrospective cohort study. Mayo Clin Proc. 2009 Dec;84(12):1073-78. [PMID 19955244]

8. You JJ, Singer DE, Howard PA, et al. Antithrombotic therapy for atrial fibrillation: Antithrombotic therapy and prevention of thrombosis, 9th ed: American College of Chest Physicians Evidence-Based Clinical Practice Guidelines. Chest. 2012 Feb;141(2 Suppl):e531s-75s. [PMID 22315271]

(C) The Internet Journal of Allied Health Sciences and Practice, 2015 
9. Holbrook A, Schulman S, Witt DM, et al. Evidence-based management of anticoagulant therapy: Antithrombotic therapy and prevention of thrombosis, 9th ed: American College of Chest Physicians Evidence-Based Clinical Practice Guidelines. Chest. 2012 Feb;141(2 Suppl):e152s-84s. [PMID 22315259]

10. Spruill WJ, Wade WE, Huckaby WG, Leslie RB. Achievement of anticoagulation by using a weight-based heparin dosing protocol for obese and nonobese patients. Am J Health Syst Pharm. 2001 Nov;58(22):2143-46. [PMID 11760916]

11. Hurewitz AN, Khan SU, Groth ML, Patrick PA, Brand DA. Dosing of unfractionated heparin in obese patients with venous thromboembolism. J Gen Intern Med. 2010 May;26(5):487-91. [PMID 21161426]

12. World Health Organization. Obesity and overweight. Updated January 2015. http://www.who.int/mediacentre/factsheets/fs311/en/ Accessed August 5, 2015.

13. Schulman S, Kearon C. Definition of major bleeding in clinical investigations of antihemostatic medicinal products in non-surgical patients. J Thromb Haemost. 2005 Apr;3(4):692-4. [PMID 15842354] 